\title{
Adsorption therapy in critically ill with septic shock and acute kidney injury: a retrospective and prospective cohort study
}

Gregor A. Schittek ${ }^{1 *} \mathbb{0}$, Philipp Zoidl ${ }^{1}$, Michael Eichinger ${ }^{1}$, Simon Orlob ${ }^{1}$, Holger Simonis ${ }^{1}$, Martin Rief $^{1}$, Philipp Metnitz ${ }^{1}$, Tobias Fellinger ${ }^{2}$ and Jens Soukup ${ }^{3}$

\begin{abstract}
Background: Haemoadsorption has been described as an effective way to control increased pro- and anti-inflammatory mediators ("cytokine storm") in septic shock patients. No prospective or randomised clinical study has yet confirmed these results. However, no study has yet prospectively specifically investigated patients in severe septic shock with sepsis-associated acute kidney injury (SA-AKI). Therefore, we aimed to examine whether haemoadsorption could influence intensive care unit (ICU) and hospital mortality in these patients. Furthermore, we examined the influence of haemoadsorption on length of stay in the ICU and therapeutic support.
\end{abstract}

Methods: Retrospective control group and prospective intervention group design in a tertiary hospital in central Europe (Germany). Intervention was the implementation of haemoadsorption for patients in septic shock with SA-AKI. 76 patients were included in this analysis.

Results: Severity of illness as depicted by APACHE II was higher in patients treated with haemoadsorption. Riskadjusted ICU mortality rates (O/E ratios) did not differ significantly between the groups ( $0.80 \mathrm{vs}$. 0.83 ). We observed in patients treated with haemoadsorption a shorter LOS and shorter therapeutic support such as catecholamine dependency and duration of RRT. However, in multivariate analysis (logistic regression for mortality, competing risk for LOS), we found no significant differences between the two groups.

Conclusions: The implementation of haemoadsorption for patients in septic shock with acute renal failure did not lead to a reduction in ICU or hospital mortality rates. Despite univariate analysis delivering some evidence for a shorter duration of ICU-related treatments in the haemoadsorption group, these results did not remain significant in multivariate analysis.

Trial registration CytoSorb ${ }^{\circledR}$ registry https://clinicaltrials.gov/ct2/show/NCT02312024. December 9, 2014. Database: https://www.cytosorb-registry.org/ (registration for content acquisition is necessary)

Keywords: Cytosorb, Haemoadsorption, Mortality, Outcome, Sepsis, Septic shock, Acute kidney injury

*Correspondence: gregor.schittek@medunigraz.at

1 Department of Anaesthesiology and Intensive Care Medicine, Division of General Anaesthesiology and Intensive Care Medicine, Medical University of Graz, Auenbruggerplatz 5, 8036 Graz, Austria

Full list of author information is available at the end of the article

\section{Introduction}

Sepsis is a life-threatening organ dysfunction caused by a dysregulated immune response after infection and is one of the worldwide leading causes of death $[1,2]$. The definition of septic shock, as a subtype of sepsis, includes circulatory instability and therefore the need for vasopressors after volume substitution as well as cellular and metabolic malfunctioning with an increased lactate level. 
Septic shock is associated with higher in-hospital mortality compared to patients that suffer from sepsis alone. Among septic shock patients, those with acute renal failure (ARF) have the highest mortality [3]. Presumably, a "cytokine storm" causes the worsening of sepsis to septic shock [4]. This hyperinflammatory reaction is not only induced by the release of endogenous cytokines (IL-1 $\beta$, IL-6, IL-12, TNF- $\alpha$ ), but also the exotoxic ones, like pattern-associated molecular patterns (PAMPs) or damageassociated molecular pattern (DAMPs).

Whole blood absorbers can remove excessive inflammatory mediators like cytokines, chemokines, haemoglobin, myoglobin, bilirubin, bile acids, bioactive lipids, light chains from antibodies, toxins, enterotoxins (Shiga toxins, alpha-haemolysin, gangrene toxins, diphtheria toxins) and other toxic metabolites [5]. The absorber can be connected to either an extracorporeal membrane oxygenation (ECMO) device or renal replacement therapy (RRT) and will partly eliminate the abovementioned mediators [6]. With regard to the current literature on haemoadsorption, it would seem reasonable and associated only with the slightest logistics to add Cytosorb ${ }^{\mathrm{TM}}$ to the RRT in septic patients with ARF for haemoadsorption. However, no study has yet prospectively analysed patients in septic shock with sepsis-associated acute kidney injury (SA-AKI) [6-19]. Until now, only case reports/ series and retrospective studies have supported in their findings the utilisation of the absorber we used in this study [6-20]. While the adsorber has not been approved by the FDA but for COVID-19 emergency treatment, the European Medicines Agency (EMA) decided to do so. Despite its approval in Europe in 2011, there still have not been any prospective studies supporting the efficacy of the absorbers. Only one randomised, controlled multicentre trial exists. In that study, the primary endpoint was a reduction in IL-6 in sepsis via absorption. Secondary endpoints were a reduction in the multiorgan failure (MOF) rate and ventilator-free days and a positive impact on oxygenation. The primary and secondary endpoints of this multicentre trial showed no impact of haemoadsorption. However, patients in severe sepsis with ARF were only a small subgroup $(N=15[32 \%]$ vs. $N=8[16 \%])$, so no analysis of patients in septic shock with ARF was possible [8].

One recent randomised-controlled single-centre pilot study described 20 patients in septic shock without ARF and showed a significant reduction in catecholamine requirements as well as PCT levels in patients who received therapy with the absorber [7]. The authors could not conduct an outcome analysis because of the small patient number.

Due to the missing evidence regarding haemoadsorption, we decided in 2015 to participate in the
CytoSorb ${ }^{\circledR}$ registry trial and further conduct a pre-post analysis. The objective of our analysis was to determine whether the implementation of haemoadsorption in septic shock patients with SA-AKI influences intensive care unit (ICU)-related outcomes. Primarily, we aimed to examine whether haemoadsorption would influence ICU mortality. Secondarily, we aimed to examine the influence on length of treatment, such as renal replacement therapy (RRT), ICU length of stay (LOS), catecholamines and duration of ventilatory support.

\section{Materials and methods}

\section{Selection and description of participants}

After obtaining approval for participation in the Cytosorb ${ }^{\circledR}$ registry by the local ethics committee [AS 88(bB)/28.01.2015, Chairman Prof. M. Matthias], we prospectively collected data in our ICU at a tertiary hospital in Cottbus, Germany, starting from 2015, for a period of 3 years [20]. We decided to utilise haemoadsorption with Cytosorb ${ }^{\mathrm{TM}}$ in all patients in the aforementioned period with following condition: septic shock (rising noradrenaline dose above $20 \mu \mathrm{g} / \mathrm{min}$.) with sepsis-associated acute kidney injury in adult patients treated in our ICU. The Cytosorb ${ }^{\circledR}$ registry was designed to explore the use of the Cytosorb ${ }^{\mathrm{TM}}$ (Cytosorbents Corp., NJ, USA) absorber in critically ill patients under real-life conditions using all relevant information (e. g., diagnosis, comorbidities, treatment/concomitant medication, clinical laboratory parameters, outcome) during haemoadsorption (ClinicalTrials.gov Identifier: NCT02312024) [20, 21]. The primary endpoint of this prospective, multicentre registry involving more than 130 centres from 22 countries was to compare the in-hospital mortality predicted according to the APACHE II score. APACHE II was calculated for the $24 \mathrm{~h}$ prior to initiation of CVVHDF/haemoadsorption (according to the registry protocol). Additionally, we retrospectively analysed identified patients' group from our ICU treated from $01 / 2012$ to $12 / 2013$ with identical clinical inclusion criteria as a historical control group with approval from the local ethics committee. In detail, inclusion criteria were septic shock (rising noradrenaline dose above $20 \mu \mathrm{g} / \mathrm{min}$.) with sepsisassociated acute kidney injury in adult patients treated in our ICU. Due to reconstruction measures and the used patient data management systems, we could only extend the historic control group back to 2012. The 3 years of prospective data recruitment were by default from the study protocol of the Cytosorb ${ }^{\circledR}$ registry. Thus our study was a single-centre prospective intervention study with historical controls. 


\section{Treatment protocol and material}

All patients were treated in accordance with the local sepsis treatment protocol, which was based on the actual guidelines valid during each treatment period [22, 23]. We evaluated the adequacy of fluid resuscitation therapy or vasopressor support using pulse indicated continuous cardiac output $\left(\mathrm{PiCCO}^{\circledR}\right.$, Getinge Deutschland $\mathrm{GmbH}$, Rastatt, Germany) or ultrasound. The initial calculated antimicrobial therapy based on the assumed or underlying septic focus according the actual hospital recommendations. The continuous veno-venous haemodiafiltration (CVVHDF, Prismaflex, M150 Filter, Fa. Baxter International Inc.) with or without haemoadsorption was used in all patients with septic shock with acute renal failure (failure stage of RIFLE criteria for 12-24 h, comparative to KDIGO stage 3 ) according to the RIFLE classification [24], increasing catecholamine dependency above a noradrenaline dose of $20 \mu \mathrm{g} / \mathrm{min}$ (despite adequate fluid resuscitation therapy) and elevated IL-6 higher than $500 \mathrm{pg} / \mathrm{ml}$. All patients were anticoagulated via the continuous administration of heparin. Thus, all patients included in our analysis presented with septic shock, an increasing catecholamine dependency above a noradrenaline dose of $20 \mu \mathrm{g} / \mathrm{min}$ and were treated with CVVHDF due to ARF.

Absorbers were changed when the CVVHDF system had to be changed or further adsorption therapy was needed. The further need for adsorption therapy was assessed whether the patient became increasingly unstable again after termination of the adsorption therapy or interleukins started increasing again.

\section{Data acquisition and statistical analysis}

Starting in January 2015, we prospectively acquired data for the Cytosorb ${ }^{\circledR}$ registry. According to the underlying protocol of the Cytosorb ${ }^{\circledR}$ registry, we chose ICU mortality as primary endpoint for our evaluation as well. Secondarily, we evaluated the hospital mortality and length of treatments in days (ICU/hospital/ventilatory support/ catecholamine therapy/CVVHDF).

Data analysis and statistics were performed with IBM SPSS Statistics 25. Data are described with median or mean, as necessary. The interquartile range (IQR) or $95 \%$ confidence interval is displayed in parentheses as appropriate. Because of the small sample size, all tests were performed as exact tests. To assess group differences, we used Levene's test and the Mann-Whitney $U$ test. We used $\alpha=5 \%$. Missing data regarded only laboratory test results, and therefore did not influence the interpretation of primary or secondary results. Significant results and differences are marked with an asterisk $(*)$ in the tables. For the purposes of this article, the term "significant" is used when data reached statistical significance, defined as $p<0.05$. Because of the missing information in the literature, no power analysis could be performed initially. Therefore, we decided to participate in the CytoSorb ${ }^{\circledR}$ registry according to protocol. The control group size was also not calculated, but we screened and evaluated all patients since the implementation of our patient data management system in 2012 until the beginning of the Cytosorb $^{\mathrm{TM}}$ implementation in 2014. To enable comparison between the groups, we calculated the observedto-expected (ICU and hospital) mortality ratio $(\mathrm{O} / \mathrm{E}$ ratio). Further analyses included logistic regression of ICU mortality and hospital mortality adjusted with the covariates "haemoadsorption", "APACHE II", "PCT prior to CVVHDF", "CRP prior to CVVHDF " and "catecholamine dosage before initiation of CVVHDF". The same covariates were used in a Fine and Gray model for the subdistribution hazard ratio of the event of "alive discharge from ICU" in presence of the competing event of death in the ICU.

\section{Results}

From $1 / 2015$ to $5 / 2018$, a total of 2,102 patients with septic shock were treated in our ICU. Of these, 844 patients developed ARF and 159 were treated with CVVHDF. We identified 43 patients fulfilling the inclusion criteria. In the historic control group (from 1/2012 to 12/2013), 672 patients with septic shock were treated in our ICU; 164 developed ARF and 70 of them were treated with CVVHDF. Furthermore, we identified 33 patients for the control group fulfilling the inclusion criteria. The characteristics of our patients (at the moment of initiation of CVVHDF or haemoadsorption) before and after the implementation of haemoadsorption were similar (see Table 1). The only difference between the groups was the significantly higher severity of illness as depicted by APACHE II $(p=0.008)$ and catecholamine dependency in patients treated with haemoadsorption $(p=0.001)$ (Table 1).

The observed ICU mortality rates of $72.1 \%$ (95\% CI 58.7-85.5\%) vs. $66.7 \%$ (95\% CI 52.6-80.8\%) were not significantly different between the groups (Table 2). When comparing risk-adjusted hospital mortality rates (observed-to-expected mortality ratios, short: $\mathrm{O} / \mathrm{E}$ ratios), no significant difference between the groups was found: 0.88 (95\% CI $0.77-0.99)$ vs. 0.80 (95\% CI 0.65-0.94). With regard to the use of haemoadsorption cartridges in survivors, approximately one cartridge per patient was utilised as the median (IQR 1,2) for $35.5 \mathrm{~h}$ $(17,47)$.

We further analysed ICU and hospital mortality by multivariate analysis (logistic regression, Additional file 1: Table S1). In both models we adjusted mortality 
Table 1 Demographics of the study population (control group vs haemoadsorption therapy)

\begin{tabular}{|c|c|c|c|}
\hline Characteristic & Control group $(n=33)$ & Haemoadsorption group $(n=43)$ & $p$ value \\
\hline Age (years) & $62(53,74)$ & $63(52,71)$ & NS \\
\hline Sex female/male & $4 / 29$ & $12 / 31$ & NS \\
\hline Weight (kg) & $87(78,105)$ & $90(75,100)$ & NS \\
\hline Septic focus & & & NS \\
\hline Abdominal & 9 & 13 & \\
\hline Catheter infection & 0 & 1 & \\
\hline CNS & 1 & 0 & \\
\hline Pulmonary & 16 & 13 & \\
\hline Soft tissue & 2 & 7 & \\
\hline Unclear & 5 & 5 & \\
\hline Urinary tract & 0 & 4 & \\
\hline CRP before CVVHDF (mg/l) ( $n=33$ vs 42$)$ & $253(173,363)$ & $250(167,337)$ & NS \\
\hline PCT before CVHDF (ng/l) & $7(2,30)$ & $28(11,67)$ & 0.002 \\
\hline IL-6 before CVHDF (pg/ml) ( $n=0$ vs 41) & Not measured & $5000(939,5000)$ & \\
\hline Noradrenaline before CVVHDF ( $\mu \mathrm{g} / \mathrm{min})$ & $44(38,62)$ & $64(48,90)$ & 0.005 \\
\hline Hydrocortisone administration & $82 \%$ & $81 \%$ & NS \\
\hline APACHE II before CVVHDF initiation & $35(33,40)$ & $39(36,42)$ & 0.01 \\
\hline Lowest MAP $24 \mathrm{~h}$ before CVHDF & $65(57,73)$ & $53(40,65)$ & 0.03 \\
\hline $\begin{array}{l}\text { Lowest GCS } 24 \mathrm{~h} \text { before CVVHDF (estimated if patient } \\
\text { already sedated) }\end{array}$ & $11(3,15)$ & $3(3,3)$ & NS \\
\hline
\end{tabular}

CNS central nervous system, CRP C-reactive protein, PCT procalcitonin, IL-6 interleukins 6, CVVHDF continuous veno-venous haemodiafiltration, MAP middle arterial blood pressure, GCS Glasgow Coma Scale, NS not significant

Table 2 Treatment and outcome of control and HA group (Cytosorb ${ }^{\mathrm{TM}}$-group)

\begin{tabular}{|c|c|c|c|}
\hline Outcome measure & Control group $(N=33)$ & Cytosorb $^{\mathrm{TM}}$-group $(N=43)$ & $p$ value \\
\hline ICU mortality rate & $66.7 \%(22 / 33)$ & $72.1 \%(31 / 43)$ & \\
\hline O/E ratio ICU mortality & $0.81(0.65-0.94)$ & $0.82(0.72-0.93)$ & \\
\hline Hospital mortality rate & $66.7 \%(22 / 33)$ & $76.7 \%(33 / 43)$ & \\
\hline O/E ratio hospital-mortality & $0.81(0.65-0.94)$ & $0.88(0.77-0.99)$ & \\
\hline LOS ICU (days [d]) & $21(6,54)$ & $12(3,23)$ & 0.026 \\
\hline LOS ICU for survivors (d) [N=11 vs 12] & $64(45,80)$ & $29(20,40)$ & 0.006 \\
\hline LOS ICU for non-survivors (d), [N=22 vs 31] & $10(4,20)$ & $7(3,14)$ & 0.115 \\
\hline LOS hospital (d) & $25(11,71)$ & $15(5,30)$ & 0.040 \\
\hline LOS hospital survivors (d) & $86(68,122)$ & $52(38,45)$ & 0.004 \\
\hline LOS hospital non-survivors (d) & $13(5,28)$ & $8(5,17)$ & 0.096 \\
\hline Length of ventilatory support (d) & $19(5,41)$ & $8(3,17)$ & 0.009 \\
\hline Length of ventilatory support for survivors (d) & $42(37,72)$ & $20(11,26)$ & 0.001 \\
\hline Length of ventilatory support non-survivors (d) & $10(4,20)$ & $6(3,13)$ & 0.121 \\
\hline Length of CVVHDF (d) & $6(3,16)$ & $3(2,7)$ & 0.012 \\
\hline Length of CVVHDF survivors (d) & $12(4,19)$ & $6(3,10)$ & 0.260 \\
\hline Length of CVVHDF non-survivors (d) & $4(2,10)$ & $2(2,4)$ & 0.047 \\
\hline Length of catecholamine administration (d) & $18(7,30)$ & $8(3,13)$ & 0.001 \\
\hline Length of catecholamine administration survivors (d) & $29(21,47)$ & $10(7,12)$ & $>0.001$ \\
\hline Length of catecholamine administration non-survivors (d) & $11(4,20)$ & $7(3,13)$ & 0.043 \\
\hline
\end{tabular}

ICU intensive care unit, CVVHDF continuous veno-venous haemodiafiltration 
with the covariates "haemoadsorption", "APACHE II", "PCT prior to CVVHDF", "CRP prior to CVVHDF " and "catecholamine dosage before initiation of CVVHDF". The coefficient for haemoadsorption was not significant in either of those models (OR ICU 0.3-3.0 $[p=0.99]$ and OR hospital 0.43-4.05 [ $p=0.64]$ ).

In univariate analysis of all patients, we found that LOS, ventilatory support, duration of CVVHDF and duration of catecholamine administration were significantly lower for patients in the haemoadsorption group $(p<0.01)$ (Table 2). These results remained significant when only survivors were analysed. We found that length of stay and treatments except for duration of CVVHDF were in favour of the haemoadsorption group (Table 2, Figs. 1, 2). Their ICU-LOS for survivors was $29(20,40)$ vs. $64(45,80)$ days $(p=0.006)$.

However, when analysing LOS for all patients with a competing risk model (Fine and Gray), where LOS was adjusted for the same covariates as mentioned for the logistic regression above, no difference between the two groups remained significant (Fig. 3).

\section{Discussion}

Our study shows that after adjustment for covariates, there was no benefit of haemoadsorption in patients with septic shock on either mortality or LOS in the ICU. Regarding LOS, we found a significant difference between the two groups only in univariate analysis, but not in multivariate analysis. It cannot be excluded that this discrepancy might be due to the small number of observations, which makes adjustment for several covariates difficult.

Although we found some evidence, that application of haemoadsorption reduced the duration of several invasive ICU-related treatments, such as RRT, ventilation and catecholamine dependency; it has to be highlighted, that despite the shorter duration, these patients did not show lower ICU or hospital mortality. Our findings are in line with the existing RCTs, where the authors stated that no mortality reduction could be found $[7,8]$. In the multicentric RCT form Schädler et al. patients with sepsis and ARDS were investigated. However, the investigated groups had far lower ARF rates (16\% vs. $32 \%)$ and APACHE II values (23-25 vs. 35-39) than our patients. Despite our matching resulting in more homogenous groups, we could only confirm their main result regarding mortality [8]. In the second RCT from Hawchar et al., only 20 patients without ARF were investigated and thus no mortality analysis was planned. In September 2019, a retrospective study assessing septic shock patients and CVVHDF was published [19]. In this publication, the authors stated that they observed a nonsignificant reduction in 28 -day mortality ( $48 \%$ vs. $51 \%$ ). After statistically adjusting their data, they found that patients treated with haemoadsorption had significantly lower 28 -day mortality ( $52 \%$ vs. $72 \%)$. Comparison of their results with our own or further studies is difficult due to the retrospective nature, lack of randomisation in treatment assignment and missing explanations as to why each treatment was chosen.

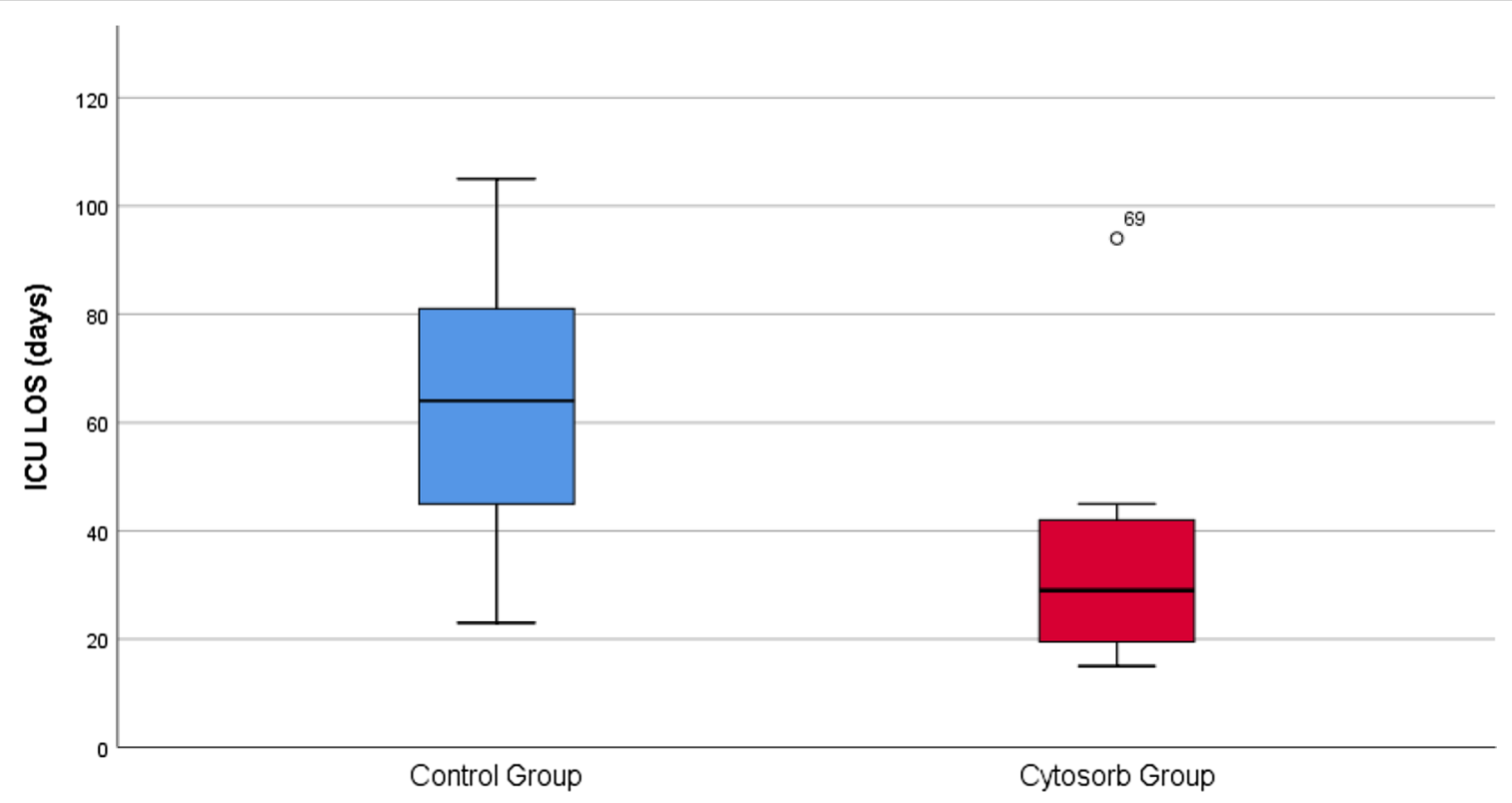

Fig. 1 Blue: control group — patients without haemoadsorption $(n=11)$; red: patients with haemoadsorption $(n=12)$ 


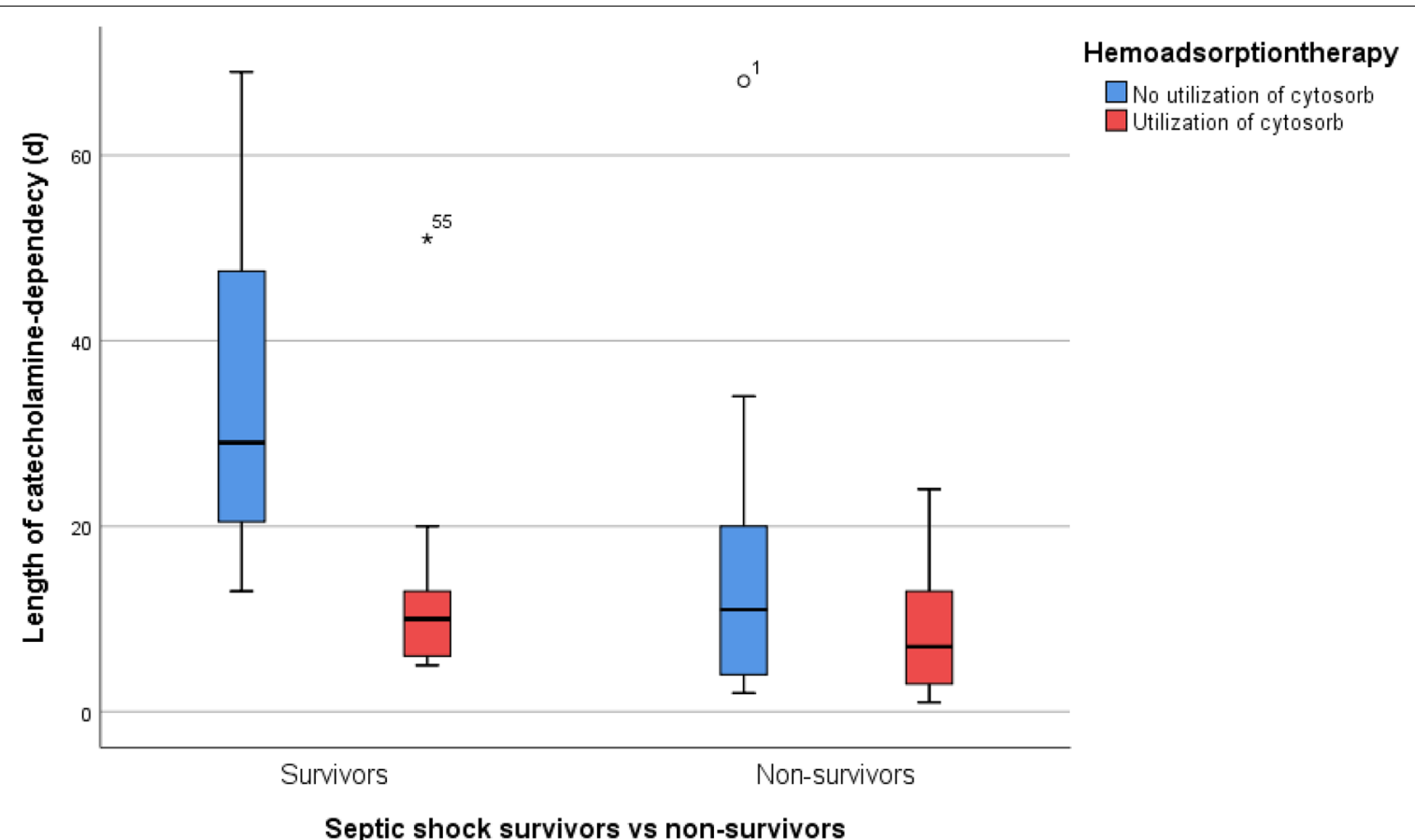

Fig. 2 ICU length of catecholamine dependency for patients without (blue) and with (red) haemoadsorption. For ICU survivors, $N=11$ (control group) vs. $N=12$ (Cytosorb $^{\mathrm{TM}}$ group). For ICU non-survivors, $N=22$ vs. $N=31$, respectively

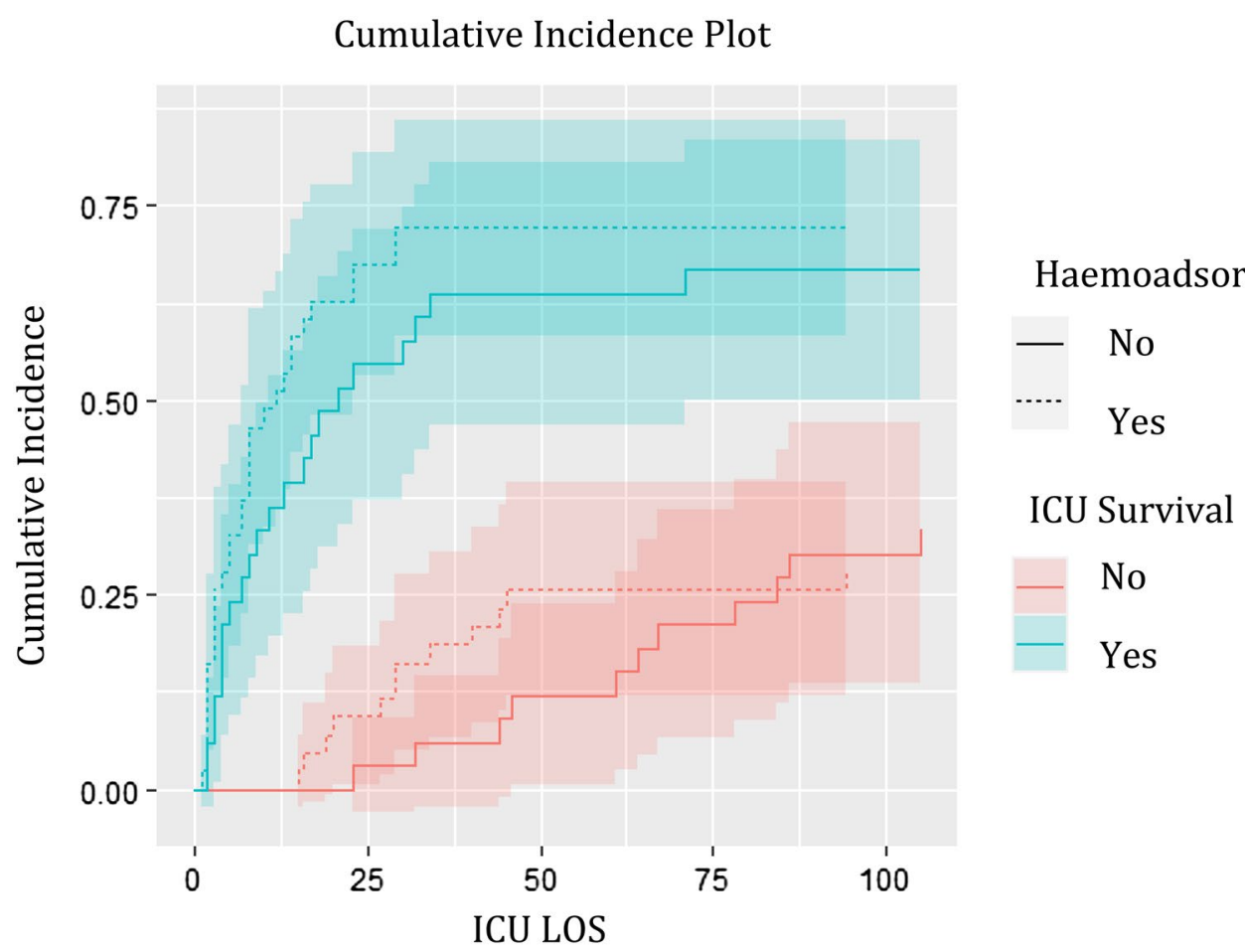

Fig. 3 Influence of haemoadsorption on the time to event for alive discharge from ICU vs. death in ICU unadjusted cumulative incidence plots. The confidence intervals overlap, showing no significant difference in the cumulative incidences 
Regarding secondary outcome analyses, we observed that after adjustment of covariates, patients with haemoadsorption did not have lower mortality rates. While Schädler et al. [8] did not evaluate treatment duration (ICU, hospital, ventilator therapy, CVVHDF), Hawchar et al. could also not find a significant difference regarding ICU-LOS in their pilot trial [7]. While the authors described no effect on mortality or LOS, they stated that that they could significantly reduce catecholamines during haemoadsorption [7]. Although these results seem to be in line with ours, their described result is only referring to the pre-post values of the haemoadsorption group and is not comparing the two groups to each other. Further, because their control group was older and had higher expected mortality ( $57 \%$ vs. $70 \%$ ) their results could just be the description of the effect of convalescence of a healthier population group, or merely be coincidence due to the small sample size.

Furthermore, we noticed that a reduction in the negative effects of the cytokine storm has also been described as an immunomodulatory effect by macrolides. In 2013, it was shown in an RCT with 600 septic patients in Greece that the i.v. administration of clarithromycin $(1 \mathrm{~g}$ daily over 4 days) reduced the treatment costs by approximately $30 \%$ [29]. In the subgroup analysis of patients with septic shock and multiorgan failure, the authors found that patients treated with additional clarithromycin had significantly lower 28-day mortality $(73.1 \%$ [19/26] vs. $53.6 \%[15 / 28])$ and shorter duration of sepsis treatment (10 vs. 6 days). Since these results are derived of a subgroup of $<10 \%$ of the total study population, they have also to be interpreted as exploratory and with caution.

The difference in the severity of illness between our two investigated groups did not introduce a bias since we compare only risk-adjusted mortality rates. However, we have to admit that APACHE II was not calculated using the worst values of the first $24 \mathrm{~h}$ after admission. We used instead the last $24 \mathrm{~h}$ before administration of haemoadsorption to calculate APACHE II values according to the registry's protocol. However, since this was done for both groups in the same way, values still remain comparable.

In addition, it has to be mentioned that although haemoadsorption was not to be considered as ultima ratio for the patients in our setting, the availability of this new technology may have encouraged clinicians to further escalate sepsis therapy for patients, which would otherwise not have been performed (e.g. CVVHDF). This would explain why more patients in the haemoadsorption group had lower MAP and GCS scores.

A limitation of our study is of course its monocentric design. This could have caused a selection bias in the study population and how the data were acquired, which may have led to results that cannot be generalised. In this context, an RCT with the primary endpoint of a reduction in ICU-related treatment duration (possibly through immunomodulation) could form a base for a further evaluation of haemoadsorption in septic shock and its efficacy as part of a sepsis treatment bundle.

\section{Conclusions}

The implementation of haemoadsorption for patients in severe septic shock with acute renal failure did not lead to a reduction in ICU or hospital mortality rates. Despite univariate analysis delivering some evidence for a shorter duration of ICU-related treatments in the haemoadsorption group, these did not remain significant in multivariate analysis. Further studies are needed to evaluate the effects of haemoadsorption in critically ill patients with severe septic shock and SA-AKI.

\section{Supplementary information}

Supplementary information accompanies this paper at https://doi. org/10.1186/s13613-020-00772-7.

Additional file 1: Table S1. Logistic regression on mortality and Fine \& Gray Model on LOS. The logistic regression model with ICU mortality and hospital mortality respectively were fitted with the covariates "haemoad sorption", "APACHE II", "PCT prior to CVHDFF", "CRP prior to CVHDF " and "catecholamine dosage before initiation of CVVHDF".. The coefficient for haemoadsorption was not significant in neither of those models. A Fine and Gray model for the subdistribution hazard ratio of the event of alive discharge from ICU in presence of the competing event of death in the ICU was fitted with the same covariates as the logistic regression models. None of the covariates showed a significant influence.

Abbreviations

APACHE II: Acute Physiology and Chronic Health Evaluation; ARF: Acute renal failure; CRP: C-reactive protein; CVVHDF: Continuous veno-venous haemodiafiltration; DAMPs: Damage-associated molecular pattern; ECMO: Extracorporeal membrane oxygenation; EMA: European Medicines Agency; FDA: Food and Drug Administration; GCS: Glasgow Coma Scale; HA: Haemoadsorption; HR: Hazard ratio; ICU: Intensive care unit; LOS: Length of stay; MAP: Mean arterial blood pressure; MOF: Multiorgan failure; O/E: Observed to expected; OR: Odds ratio; PAMPs: Pattern-associated molecular patterns; PCT: Procalcitonin; RCT: Randomised controlled trial; RRT: Renal replacement therapy; SA-AKI: Sepsis associated acute kidney injury.

\section{Acknowledgements}

A senior researcher of the Institute for Medical Informatics, Statistics and Documentation (Gerold Schwantzer) checked the correctness of the performed analysis. The team of the Centre for Clinical Studies at Jena University Hospital provided access and organised the Cytosorb ${ }^{\mathrm{TM}}$ register.

\section{Authors' contributions}

Contributed to conception and design: GAS. Contributed to acquisition of data: GAS, JS. Contributed to analysis of data: all authors. Contributed to interpretation of data: all authors. Drafting the work: GAS. Revising the paper for important intellectual content: all authors. Final approval of the submitted version: all authors. Agreement to be accountable for all aspects of the work in ensuring that questions related to the accuracy or integrity of any part of the work are appropriately investigated and resolved: all authors. All authors read and approved the final manuscript. 


\section{Funding}

None for our part; the CytoSorb ${ }^{\circledR}$ registry is organised by the Centre for Clinical Studies at Jena University Hospital and the registry is funded by CytoSorbents Europe $\mathrm{GmbH}$.

\section{Availability of data and materials}

On demand and additionally via the Cytosorb $^{\mathrm{TM}}$ register.

\section{Ethics approval and consent to participate}

The Cytosorb ${ }^{\mathrm{TM}}$ register was approved by the by the medical ethics committee of Jena and Brandenburg [AS 88(bB)/2015] in 2015. The local ethics committee did not feel compelled to make an extra vote regarding the retrospective analysis of the historical control group (decision letter 13th November 2019).

\section{Informed consent}

Informed consent ahead of recruitment was not possible.

\section{Consent for publication}

No participant-specific data are published.

\section{Competing interests}

GAS has received financial travel support for attending a symposium organised by CytoSorbents Europe GmbH in 2016. All other authors declare that there are no potential conflicts of interest to disclose.

\section{Author details}

${ }^{1}$ Department of Anaesthesiology and Intensive Care Medicine, Division of General Anaesthesiology and Intensive Care Medicine, Medical University of Graz, Auenbruggerplatz 5, 8036 Graz, Austria. ${ }^{2}$ Austrian Centre for Documentation and Quality Assurance in Intensive Care, Vienna, Austria. ${ }^{3}$ Department of Anaesthesiology, Intensive and Palliative Care, Carl-Thiem-Hospital Cottbus, Cottbus, Germany.

Received: 6 July 2020 Accepted: 7 November 2020

Published online: 18 November 2020

\section{References}

1. Fleischmann C, Scherag A, Adhikari NK, et al. Assessment of global incidence and mortality of hospital-treated sepsis. Current estimates and limitations. Am J Respir Crit Care Med. 2016;193(3):259-72. https:// doi.org/10.1164/rccm.201504-07810C.

2. Singer $M$, Deutschman CS, Seymour CW, et al. The third international consensus definitions for sepsis and septic shock (Sepsis-3). JAMA. 2016;315(8):801-10. https://doi.org/10.1001/jama.2016.0287.

3. Hoste EA, Bagshaw SM, Bellomo R, et al. Epidemiology of acute kidney injury in critically ill patients: the multinational AKI-EPI study. Intensive Care Med. 2015;41:1411-23. https://doi.org/10.1007/s0013 4-015-3934-7.

4. Weaver LK, Behrens EM. Weathering the storm: Improving therapeutic interventions for cytokine storm syndromes by targeting disease pathogenesis. Curr Treat Opt Rheumatol. 2017;3:33-48.

5. https://cytosorb-therapy.com/en/the-therapy/therapeutic-effects/. Accessed 01 Jan 2020.

6. Hetz H, Berger R, Recknagel P, et al. Septic shock secondary to $\beta$-hemolytic streptococcus-induced necrotizing fasciitis treated with a novel cytokine adsorption therapy. Int J Artif Organs. 2014;37(5):422-6. https://doi.org/10.5301/ijao.5000315.

7. Hawchar F, László I, Öveges N, et al. Extracorporeal cytokine adsorption in septic shock: a proof of concept randomized, controlled pilot study. J Crit Care. 2019:49:172-8.

8. Schädler D, Pausch C, Heise D, et al. The effect of a novel extracorporeal cytokine hemoadsorption device on IL-6 elimination in septic patients: a randomized controlled trial. PLoS ONE. 2017;12(10):e0187015. https ://doi.org/10.1371/journal.pone.0187015 (eCollection 2017).

9. Tomescu D, Dima SO, Tănăsescu S, et al. Effects of a novel cytokine haemoadsorbtion system on inflammatory response in septic shock after cephalic pancreatectomy. Rom J Anaesth Intensive Care. 2014:21(2):134-8.
10. Basu R, Pathak S, Goyal J, et al. Use of a novel hemoadsorption device for cytokine removal as adjuvant therapy in a patient with septic shock with multi-organ dysfunction: a case study. Indian J Crit Care Med. 2014;18(12):822-4. https://doi.org/10.4103/0972-5229.146321.

11. Morris C, Gray L, Giovannelli M. Early report: the use of CytosorbTM haemabsorption column as an adjunct in managing severe sepsis: initial experiences, review and recommendations. J Intensive Care Soc. 2015;16(3):257-64. https://doi.org/10.1177/1751143715574855 (Epub 2015 Mar 18).

12. Hinz B, Jauch $O$, Noky T, et al. CytoSorb, a novel therapeutic approach for patients with septic shock: a case report. Int J Artif Organs. 2015;38(8):461-4. https://doi.org/10.5301/ijao.5000429 (Epub 2015 Aug 31).

13. Houschyar KS, Nietzschmann I, Siemers F. The use of a cytokine adsorber (CytoSorb) in a patient with septic shock and multi-organ dysfunction (MODS) after a severe burn injury. Handchir Mikrochir Plast Chir. 2017;49(2):123-6. https://doi.org/10.1055/s-0042-111965.

14. Kogelmann K, Jarczak D, Scheller M, et al. Hemoadsorption by CytoSorb in septic patients: a case series. Crit Care. 2017;21(1):74.

15. Kousoulas L, Wittel U, Fichtner-Feigl S, et al. Hemoadsorption in a case of severe septic shock and necrotizing fasciitis caused by nontraumatic renal rupture due to pyelonephritis with obstructive uropathy. Case Rep Crit Care. 2018;2018:5248901. https://doi.org/10.1155/2018/52489 01.

16. Calabrò MG, Febres D, Recca G, et al. Blood purification with CytoSorb in critically ill patients: single-center preliminary experience. Artif Organs. 2019;43(2):189-94.

17. Saparov A, Sazonov V, Tobylbaeva Z, et al. First successful hemoadsorption using CytoSorb ${ }^{\circledR}$ in a septic pediatric patient in Kazakhstan: a case report. Int J Artif Organs. 2019;42(6):315-7. https://doi. org/10.1177/0391398818819953.

18. Tomescu D, Popescu M, David C, et al. Clinical effects of hemoadsorption with CytoSorb ${ }^{\circledR}$ in patients with severe acute pancreatitis: a case series. Int J Artif Organs. 2019;42(4):190-3. https://doi. org/10.1177/0391398818823762.

19. Brouwer WP, Duran S, Kuijper M, et al. Hemoadsorption with CytoSorb shows a decreased observed versus expected 28-day all-cause mortality in ICU patients with septic shock: a propensity-score-weighted retrospective study. Crit Care. 2019;23:317. https://doi.org/10.1186/ s13054-019-2588-1.

20. Friesecke S, Träger K, Schittek GA, et al. International registry on the use of the CytoSorb ${ }^{\circledR}$ adsorber in ICU patients: study protocol and preliminary results. Med Klin Intensivmed Notfmed. 2019;114(8):699-707. https://doi.org/10.1007/s00063-017-0342-5 (Epub 2017 Sep 4).

21. https://www.cytosorb-registry.org/. Accessed 01 Jan 2020.

22. Dellinger RP, Levy MM, Rhodes A, et al. Surviving sepsis campaign guidelines committee including the pediatric subgroup Surviving sepsis campaign: international guidelines for management of severe sepsis and septic shock, 2012. Intensive Care Med. 2013;39(2):165-228.

23. Rhodes A, Evans LE, Alhazzani W, et al. Surviving sepsis campaign: international guidelines for management of sepsis and septic shock: 2016. Intensive Care Med. 2017;43(3):304-77.

24. Bellomo R, Ronco C, Kellum JA, Palevsky P and the ADQI workgroup, et al. Acute renal failure-definition, outcome measures, animal models, fluid therapy and information technology needs: the Second International Consensus Conference of the Acute Dialysis Quality Initiative (ADQI) Group. Crit Care. 2004;8:R204.

25. Sandiumenge A, Diaz E, Bodi M, et al. Therapy of ventilator-associated pneumonia. A patient-based approach based on the ten rules of "The Tarragona Strategy." Intensive Care Med. 2003;29(6):876-83. https://doi. org/10.1007/s00134-003-1715-1 (Epub 2003 Apr 2).

26. Gruda MC, Ruggeberg KG, O'Sullivan P, et al. Broad adsorption of sepsis-related PAMP and DAMP molecules, mycotoxins, and cytokines from whole blood using CytoSorb ${ }^{\circledR}$ sorbent porous polymer beads. PLoS ONE. 2018;13(1):e0191676. https://doi.org/10.1371/journ al.pone.0191676.

27. Malard B, Lambert C, Kellum JA. In vitro comparison of the adsorption of inflammatory mediators by blood purification devices. Intensive Care Med Exp. 2018;6(1):12. https://doi.org/10.1186/s4063 5-018-0177-2. 
28. Honore PM, Hoste E, Molnár Z, et al. Cytokine removal in human septic shock: where are we and where are we going? Ann Intensive Care. 2019;9(1):56. https://doi.org/10.1186/s13613-019-0530-y.

29. Giamarellos-Bourboulis EJ, Mylona V, Antonopoulou A, et al. Effect of clarithromycin in patients with suspected Gram-negative sepsis: results of a randomized controlled trial. J Antimicrob Chemother. 2013;69(4):11118. https://doi.org/10.1093/jac/dkt475.

\section{Publisher's Note}

Springer Nature remains neutral with regard to jurisdictional claims in published maps and institutional affiliations.

\section{Submit your manuscript to a SpringerOpen ${ }^{\circ}$ journal and benefit from:}

- Convenient online submission

- Rigorous peer review

- Open access: articles freely available online

- High visibility within the field

- Retaining the copyright to your article

Submit your next manuscript at $\boldsymbol{\nabla}$ springeropen.com 